\title{
Desarrollo de aplicación móvil para generar competencias en los alumnos de la Universidad Tecnológica de San Juan del Río
}

\section{Development of mobile application to generate competences in the students of the Technological University of San Juan del Río}

\author{
VALENCIA-GARCÍA, Alejandro César†, GONZÁLEZ-MARTÍNEZ, Carlos Daniel y \\ BUSTAMANTE-HERNÁNDEZ, José Alberto
}

Universidad Tecnológica de San Juan del Río

ID 1 ${ }^{\text {er }}$ Autor: Alejandro César, Valencia-García / ORC ID: 0000-0002-6671-7915

ID $1^{\text {er }}$ Coautor: Carlos Daniel, González-Martínez / ORC ID: 0000-0002-7126-8683

ID $2^{\text {do }}$ Coautor: José Alberto, Bustamante-Hernández / ORC ID: 0000-0003-1210-9460

DOI: $10.35429 / J I T C .2019 .10 .3 .1 .6$

Recibido 06 de Septiembre, 2019; Aceptado 20 de Diciembre, 2019

\section{Resumen}

Estadísticas nos dicen que a finales del año 2017 se tiene un registro de aproximadamente 8 millones de aplicaciones en Google Play, Android en el 2016 mostró un total de descargas de aplicaciones de 90 mil millones; por lo tanto, los usuarios utilizan un promedio de 9 aplicaciones diarias y 30 aplicaciones mensuales. Con las anteriores estadísticas, se realizó el desarrollo de una Aplicación Móvil en Android, la cual consiste en generar preguntas aleatoriamente mediante la selección de un grado con sus respectivas opciones de respuesta; si es correcta se otorgan 5 puntos, por lo contrario se restará 3. La metodología aplicada fue SCRUM, el proyecto se realizó en 5 fases las cuales fueron: reunión de planificación, scrum diario, trabajo de desarrollo durante el sprint, revisión del sprint y retrospectiva del sprint. Se desarrolló con lenguajes de programación JSON, JAVA, PHP e integración de base de datos MySQL. Al final obtuvimos un apk que puede ser utilizada en cualquier dispositivo con Android 7.0 y superiores.

Aplicación Móvil, Acertijo, Tecnología, Android, Scrum.

\begin{abstract}
Statistics tell us that at the end of 2017 there is a record of approximately 8 million applications on Google Play, Android in 2016 showed a total of downloads of applications of 90 billion; therefore users use an average of 9 applications per day and 30 applications per month. With the above statistics, the development of a Mobile Application on Android was carried out, which consists of randomly generating questions by selecting a grade with their respective response options, if correct, 5 points are awarded, otherwise 3 will be subtracted. The methodology applied was SCRUM, the project was carried out in 5 phases which were: planning meeting, daily scrum, development work during the sprint, sprint review and sprint retrospective. It was developed with programming languages JSON, JAVA, PHP and SQL database integration. In the end we got an apk that can be used on any device with Android 7.0 and above.
\end{abstract}

Mobile Application, Quiz, Technology, Android, Scrum

Citación: VALENCIA-GARCÍA, Alejandro César, GONZÁLEZ-MARTíNEZ, Carlos Daniel y BUSTAMANTEHERNÁNDEZ, José Alberto. Desarrollo de aplicación móvil para generar competencias en los alumnos de la Universidad Tecnológica de San Juan del Río. Revista de Tecnologías de la Información y Comunicaciones. 2019. 3-10: 1-6

\footnotetext{
$\dagger$ Investigador contribuyendo como primer autor.
} 


\section{Introducción}

Hoy en día se han generado varias formas o técnicas de estudio ya que como todo se va actualizando también se deben de actualizar las formas de aprender.

Un quiz es una prueba rápida que se usa mucho a nivel escolar. Son pequeñas pruebas, pero son esenciales para preparar a los estudiantes para las pruebas más amplias y complicadas.

Comúnmente pueden tener la forma de un juego, al estilo de esos juegos de trivias en los que se selecciona la respuesta correcta entre múltiples opciones. Los quizzes también se realizan en competiciones, en las cuales los participantes deben responder rápidamente a las preguntas. (Vaivasuata, 2014)

Con este tipo de evaluaciones rápidas decidimos combinar lo que es muy utilizado hoy en día, como es el Smartphone, ya que la mayoría de las personas pasamos gran parte de nuestro tiempo revisando aplicaciones.

Decidimos desarrollar una App para la Universidad Tecnológica de San Juan del Río, la cual está desarrollada con Java, en conjunto con MySQL, PHP, JSON y un almacenamiento en host, ya que funciona mediante la conexión a Internet, y respectivamente es implementada en el sistema Android con la versión correspondiente a 7.0 y superiores (no será compatible con versiones antiguas de Android).

Para el desarrollo del proyecto Quiz Pop se implementó la metodología Scrum con la cual se implementaron 5 fases las cuales fueron: reunión de planificación, scrum diario, trabajo de desarrollo durante el sprint, revisión del sprint y retrospectiva del sprint.

En clases de la universidad se nos pidió generar un proyecto en el cual integráramos todas las habilidades adquiridas durante este tiempo. Por lo cual nos dimos a la tarea de observar lo que haría falta en la universidad ya que, en ese momento, había un gran desaprovechamiento escolar, ya que los alumnos se la pasan mirando sus teléfonos o haciendo otras cosas en vez de poner atención en clase. Por eso mismo creamos una aplicación la cual nos permitiría estudiar o reforzar los conocimientos aprendidos en clase, mediante preguntas generadas aleatoriamente.

\section{Marco Teórico}

Revisando algunos artículos sobre el aprovechamiento escolar de hoy en día, que hablaban sobre la importancia de las nuevas tecnologías, ya que las generaciones están cambiando, y como esto podía influir en el comportamiento académico de los alumnos.

Nos dimos a la tarea de formular la siguiente hipótesis, la forma tradicional de cómo se está llevando a cabo la enseñanza en algunos casos pierde interés por parte del alumno ya que en varias materias se relacionan solo con teoría, por eso para algunos alumnos es tedioso y/o aburrido.

Así que partiendo del punto anterior decidimos buscar aplicaciones similares con las cual pudiésemos tomar un ejemplo de las funciones que podría a llegar a tener nuestra aplicación, lamentablemente no encontramos muchas que nos llamaran la atención, para esto nos basamos en la aplicación de Preguntados la cual genera preguntas de cultura general, deportes, ciencia etc.

A continuación, mencionaremos los conceptos que apoyan al desarrollo de la aplicación, así como mencionar la aplicación existente mencionada anteriormente, que dieron pie a interesarnos en el proyecto.

\section{Preguntados}

Preguntados es un juego de preguntas y respuestas en el que puedes competir tanto con tus amigos como con otros miembros de nuestra comunidad. Además, puedes colaborar sugiriendo preguntas que pueden ingresar al juego si son aprobadas por la comunidad. Hay seis categorías de preguntas, estas son Historia, Ciencia, Geografía, Arte, Entretenimiento y Deporte. (Etermax, 2019)

\section{JAVA}

Java es un lenguaje de programación y una plataforma informática comercializada por primera vez en 1995 por Sun Microsystems. Hay muchas aplicaciones $y$ sitios web que no funcionarán a menos que tenga Java instalado y cada día se crean más. Java es rápido, seguro y fiable. Desde portátiles hasta centros de datos, desde consolas para juegos hasta súper computadoras, desde teléfonos móviles hasta Internet (Java, 2019)

VALENCIA-GARCÍA, Alejandro César, GONZÁLEZ-MARTÍNEZ, Carlos Daniel y BUSTAMANTE-HERNÁNDEZ, José Alberto. Desarrollo de aplicación móvil para generar competencias en los alumnos de la Universidad Tecnológica de San Juan del Río. Revista de Tecnologías de la Información y Comunicaciones. 2019 


\section{ANDROID STUDIO}

Android Studio es el entorno de desarrollo integrado (IDE) oficial para el desarrollo de aplicaciones para Android y se basa en IntelliJ IDEA. Además del potente editor de códigos y las herramientas para desarrolladores de IntelliJ, Android Studio ofrece aún más funciones que aumentan tu productividad durante la compilación de apps para Android. (Android, 2019)

\section{PHP}

Hypertext Preprocessor es un lenguaje de código abierto muy popular especialmente adecuado para el desarrollo web y que puede ser incrustado en HTML. Lo que distingue a PHP de algo del lado del cliente como JavaScript es que el código es ejecutado en el servidor, generando HTML y enviándolo al cliente. (PHP, 2019)

\section{JSON}

JavaScript Object Notation es un formato de texto que es completamente independiente del lenguaje, pero utiliza convenciones que son ampliamente conocidos por los programadores de la familia de lenguajes $\mathrm{C}$, incluyendo $\mathrm{C}, \mathrm{C}++$, C\#, Java, JavaScript y muchos otros. Estas propiedades hacen que JSON sea un lenguaje ideal para el intercambio de datos. (JSON, 2019)

\section{Desarrollo}

La idea de desarrollar una aplicación de preguntas fue en base al ya existente juego de preguntados, que consiste en arrojar preguntas de distintos temas. QuizPop tiene la misma temática pero enfocada al estudio y el aprendizaje.

Se creó primeramente en base a las materias de la carrera Tecnologías de la Información en sus 9 cuatrimestres de clases. Se tiene planeado cubrir cada una de las carreras que ofrece la Universidad Tecnológica de San Juan del Río para que todos los alumnos puedan tener una manera diferente de reforzar sus conocimientos.

Para la planeación del proyecto, se utilizó la metodología SCRUM por su flexibilidad y fácil entendimiento, para la parte técnica se utilizó el IDE Android Studio, que permite realizar aplicaciones para dispositivos Android de manera rápido y eficaz.

\section{Resultados}

La aplicación en su primera versión cubrió con los cuatrimestres de 1ro a 5to correspondientes de TSU, y de 7 mo a 9 no correspondientes a la etapa de Ingeniería de la carrera de TIC.

La aplicación cuenta con distintas funciones que ayudan a que el alumno se sienta más atraído por la aplicación, dichas funciones son:

Pantalla Principal: nos muestra el botón de iniciar sesión y la opción de empezar a jugar

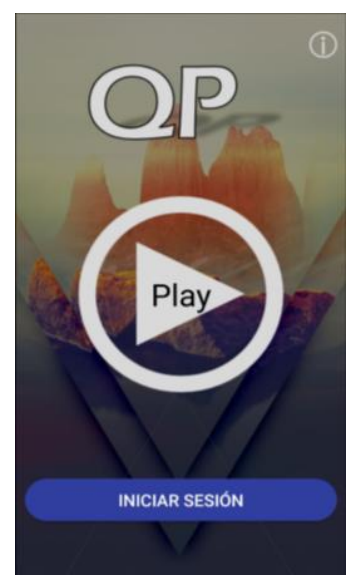

Figura 1 Pantalla principal

Fuente: Elaboración Propia

Juega Invitado: Pantalla en donde nos indica que somos invitados o básicamente que no tenemos una cuenta.

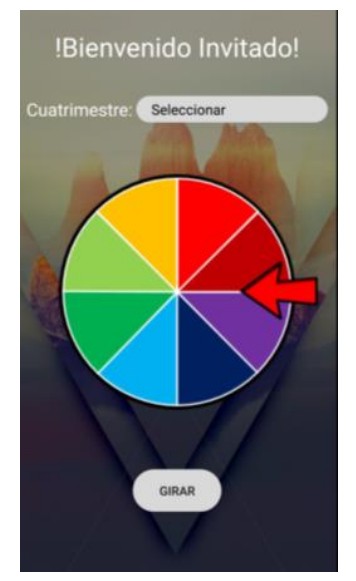

Figura2 Pantalla de invitado sin las funciones de perfil y sin poder el top10

Fuente: Elaboración Propia 
Login: Cada alumno puede registrar su número de expediente que le otorga la universidad y una contraseña, esto para guardar sus resultados.

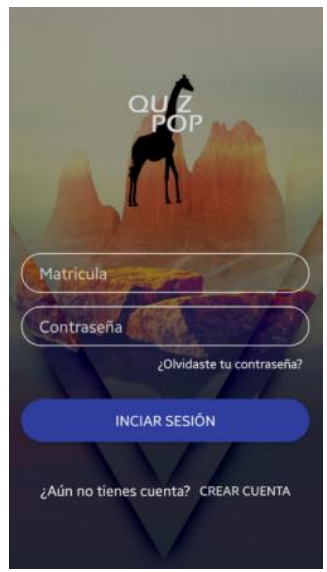

Figura 3 Captura de pantalla del login. Fuente: Elaboración Propia

Regístrate: Las personas podrán registrarse para tener un perfil y generar sus puntajes.

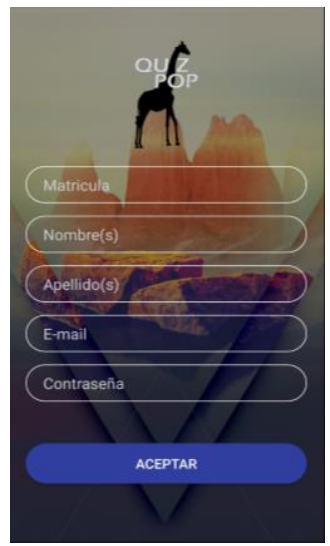

Figura 4 Captura de pantalla crear cuenta.

Fuente: Elaboración Propia

Menú: Una vez que ingresas a la te muestra las opciones complementarias perfil y puntajes.

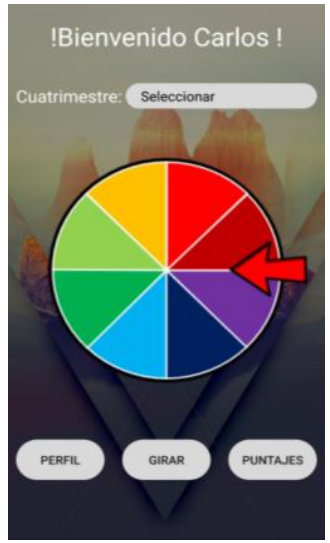

Figura 5 Captura de pantalla menú principal Fuente: Elaboración Propia
Perfil: Se muestra la información del usuario en esta pantalla con la opción de modificar sus datos y eliminar su cuenta.

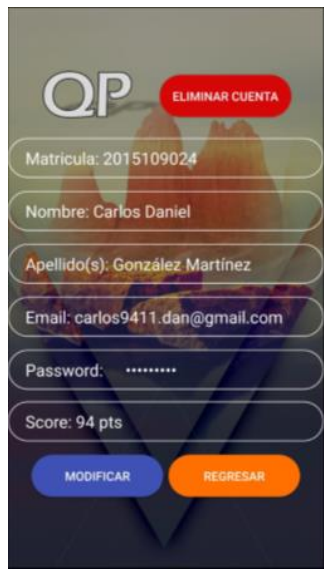

Figura 6 Captura de pantalla del perfil. Fuente: Elaboración Propia

Modifica Datos: Para cambiar nuestros datos se nos da el formulario y para confirmar el cambio de datos se pide tu contraseña, así tus amigos no la podrán cambiar.

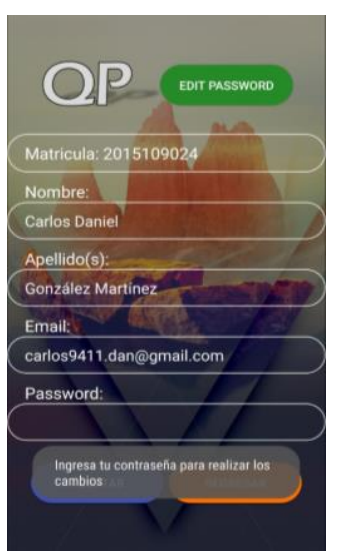

Figura7 Captura de pantalla actualización de datos Fuente: Elaboración Propia

Cambio Contraseña: Para poder cambiar la contraseña es necesario poner la anterior y que verificarla una segunda vez.

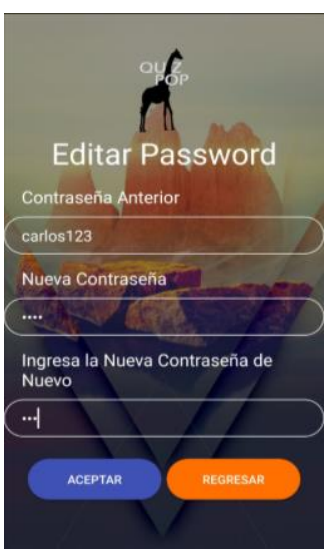

Figura 8 Captura de pantalla del login Fuente: Elaboración Propia

VALENCIA-GARCÍA, Alejandro César, GONZÁLEZ-MARTÍNEZ, Carlos Daniel y BUSTAMANTE-HERNÁNDEZ, José Alberto Desarrollo de aplicación móvil para generar competencias en los alumnos de la Universidad aplicación móvil para generar competencias en los alumnos de la Universidad
Tecnológica de San Juan del Río. Revista de Tecnologías de la Información y Comunicaciones. 2019 
Tabla de posiciones: Un apartado que muestra los puntajes de los alumnos y la posición en la que se encuentra, generando un ambiente de competitividad, esto ayudará a que se esfuercen más en alcanzar puntajes alto y a su vez reforzar sus conocimientos.

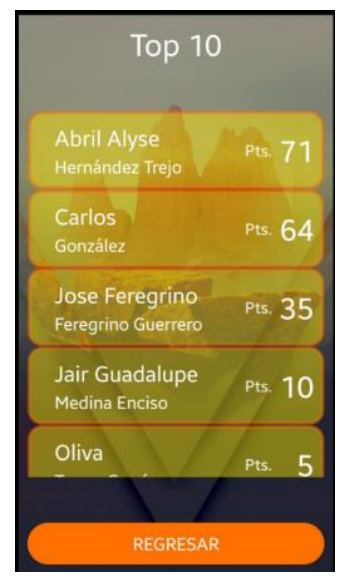

Figura 9 Captura de pantalla de tabla de posiciones Fuente: Elaboración Propia

Selección de cuatrimestres: El alumno puede elegir entre todos los cuatrimestres disponibles sin ninguna restricción para probar o reforzar sus conocimientos.

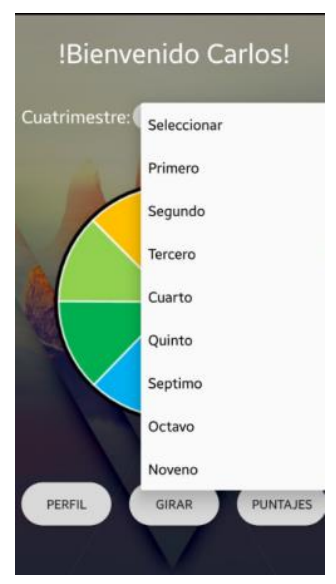

Figura 10 Captura de pantalla del menú principal (elección de cuatrimestre)

Fuente: Elaboración Propia

Preguntas al azar: Cada cuatrimestre cuenta con distintas materias y a su vez con distintas preguntas, estas se muestran de manera aleatoria y con un límite de tiempo para poder responderlas.

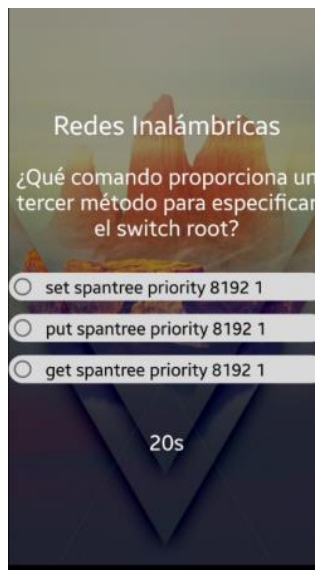

Figura 11 Captura de pantalla pregunta otorgada con respectivas respuestas y un temporizador

Fuente: Elaboración Propia

Respuesta Correcta: Cuando una respuesta es correcta, la respuesta seleccionada se pone en color verde con un mensaje que confirma que la respuesta está bien.

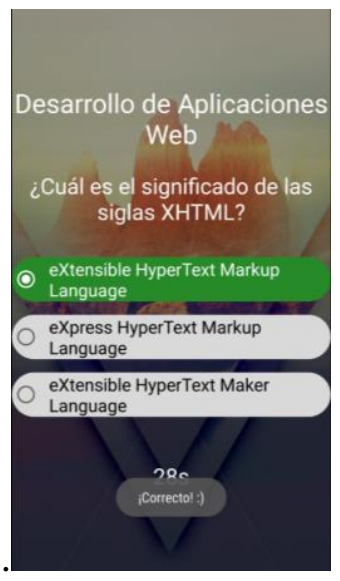

Figura 12 Captura de pantalla respuesta correcta Fuente: Elaboración Propia

Respuesta Errónea: Cuando la respuesta es incorrecta, la respuesta seleccionada se torna de color rojo, y lanza un mensaje que nos dice que la respuesta fue equivocada.

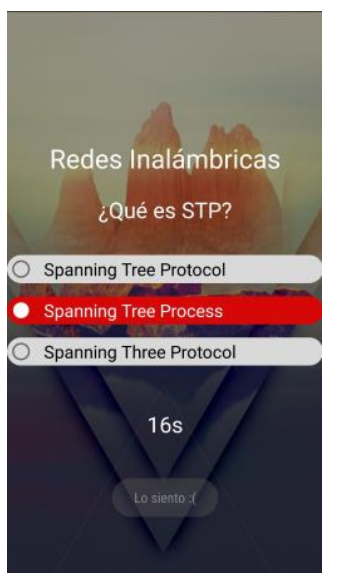

Figura 13 Captura de pantalla respuesta incorrecta Fuente: Elaboración Propia 


\section{Conclusiones}

QuizPop es un proyecto ambicioso que servirá como una herramienta para la comunidad estudiantil de la Universidad Tecnológica de San Juan del Río, ayudándolos a mejorar sus conocimientos pero de una manera más divertida y ágil. Por el momento solo se realizó una primera versión enfocada a la carrera de TIC pero planeada para todas las carreras disponibles.

\section{Referencias}

Android, D. (17 de Enero de 2019). Developers. Obtenido de https://developer.android.com/studio/intro/?hl= es-419

Etermax. (21 de Enero de 2019). Etermax. Obtenido de https://supportp2.etermax.com/customer/es/port al/articles/2525312-\%C2\%BFqu\%C3\%A9-espreguntados-?b_id=10462

Java. (21 de Enero de 2019). JAVA. Obtenido de https://www.java.com/es/download/faq/whatis_ java.xml

JSON. (22 de Enero de 2019). Obtenido de JSON: https://www.json.org/json-es.html

PHP. (22 de Enero de 2019). PHP. Obtenido de http://php.net/manual/es/intro-whatis.php

Vaivasuata. (29 de Julio de 2014). Diferencientre. Obtenido de http://diferenciaentre.info/diferencia-entre-quizy-test/ 